VOL. 7 (1972), 77-89.

\title{
Three-dimensional Stokes flow
}

\section{J.J. Mahony}

\begin{abstract}
A method is present for simplifying the calculation of steady Stokes flows. It is applicable for domains which are essentially half spaces and utilizes a special vector stream potential to decouple the boundary conditions. The results are applied to estimate the effect of no-shear zones on a shear flow.
\end{abstract}

\section{Introduction}

Despite the linear nature of the equations and apparently simple boundary conditions, the determination of the steady flow of a viscous fluid, when the Reynolds number is small, can be surprisingly difficult. Although the velocity components satisfy the biharmonic equation there are usually insufficient boundary conditions available to determine the velocity field from that equation directly. Instead there are subsidiary conditions, arising from the equation of continuity and the need for compatibility between the velocity components and the harmonic pressure field, which serve to compensate for the shortage of boundary conditions for the biharmonic equations. For flow fields which are either two-dimensional or possess axi-symmetry, the difficulties can be overcome by working in terms of suitable stream functions which satisfy fourth order partial differential equations and normal boundary conditions. For three-dimensional flow fields, for which this simplification is not available, there are very few solutions available. In Lamb [3] there are some solutions described which are obtained by semi-inverse methods, and they appear to offer little guidance as to how to solve other specific

Received 10 February 1972. This work was carried out while the author was a visitor at the CSIRO Division of Environmental i.jechanics at Canberra, and he wishes to acknowledge the hospitality of that institution. 
problems. There is a possible alternative approach based on the use of a fundamental solution which also guarantees the satisfaction of the associated compatibility conditions. This can be used to generate three singular integral equations which are normally coupled, from which in principle the solution may be computed. This integral equation formulation has been used by Williams [6] as a basis for deriving asymptotic results but it does not appeal as a practical basis for solving problems.

Some problems involving three-dimensional stokes flows have arisen recently in connection with the effect of fluid-fluid interfaces on the resistance to flow in a porous medium. The interaction of such interfaces depends on the cleanliness of the surface which could thus affect the hydraulic conductivity of the medium. Recently Philip [5] has contended that lack of ability to support shear stress on such interfaces would have only very localized effects. To support this contention he calculated the effects on certain basic shear flows of arrangements of one or more strips on the boundary where no shear stress could be supported. Such geometry is not a realistic model for the interfacial problem of the flow in a porous medium, and so some assessment of the relative magnitude of two- and threedimensional geometries was sought.

The problem which was considered initially was the effect of a circular no-shear zone in a rigid plane bounding a parallel shear flow. However the technique which led to the solution appears to have wider applications. Thus in Section 2 of this paper there is a general discussion of a method for reducing a range of Stokes flows to standard boundary value problems. This is achieved by the use of a specially chosen vector stream function which, while not as powerful as the scalar stream function in two-dimensional flows, does reduce the magnitude of the problem. Normally the reduced problem involves the determination of two biharmonic functions and one harmonic function subject to standard boundary conditions. There are cases where the problems can be reduced still further and a no-shear zone happens to be one of these. In Section 3 this method is applied to the problem described at the beginning of this paragraph. From this solution estimates are obtained which enable comparisons to be made between two- and three-dimensional no-shear zones which occupy the same fraction of the area of the rigid surface. It is shown that there is a much smaller effect on the basic shear flow when the 
no-shear zone is localized. It seems indicated that the estimates used by Philip [5] are high and that the interfacial actions have even smaller effects than he has suggested.

2. Reduction to standard boundary value problems

The equations governing the Stokes flow of a viscous flow are the momentum equation

$$
\mu \nabla^{2} u=\nabla p
$$

and the equation of continuity

$$
\nabla \cdot u=0
$$

Here $\mu$ is the viscosity of the fluid, $u=(u, v, w)$ its velocity and $p$ is the presure at the point with coordinates $(x, y, z)$ relative to some rectangular cartesian axes. These equations provide an adequate description of the flow field for a Newtonian fluid only when the relevant Reynolds number is small. In an unbounded domain the equation ( 1 ) is not generally adequate for the far flow field but for three-dimensional flows due to bounded disturbing influences this does not have serious implications for the determination of the near flow field using the above equations. It will be assumed subsequently that the solution will be used only at distances from the disturbance such that the Reynolds number, based on any such distance, is small. In this paper interest is centred on domains of flow which approximately comprise the half space $z>0$ or whose discussion can be reduced to that of such a domain by symmetry considerations. The approximate half space is defined by $z>Z(x, y)$ where $Z$ is a prescribed function which vanishes outside some finite domain in the $(x, y)$-space. The methods can be extended to deal with domains approximating, in the above sense, the strip $0<z<d$ where $d$ is some fixed constant; but the details are not presented here. Further it will be assumed that the velocity field vanishes at infinity. This is not a serious restriction because the linear nature of the problem enables one to add an appropriate velocity field which satisfies all the conditions save those which contribute to the disturbance which are merely altered in a calculable fashion.

It may be shown that equations (1) and (2) imply 


$$
\begin{aligned}
& \nabla^{2} p=0, \\
& \nabla^{4} u=0,
\end{aligned}
$$

but the boundary conditions are rarely of a form which permit the pressure of any of the velocity components to be deduced from these equations. However the equation of continuity implies that the velocity field is derivable as the curl of a vector stream potential, which however is unique only up to an arbitrary gradient function. For two-dimensional flows the introduction of the stream function reduces the number of functions needed to describe the velocity field. In three-dimensional flows an arbitrary choice of a vector stream potential does not yield such a simplification, but recently Benjamin and Mahony [1] have utilized a particular vector potential $A=(A, B, 0)$ which does have this result. In the present context the components $A$ and $B$ may be defined by

$$
A(x, y, z)=-\int_{z}^{\infty} v\left(x, y, z^{\prime}\right) d z^{\prime}
$$

and

$$
B(x, y, z)=\int_{z}^{\infty} u\left(x, y, z^{\prime}\right) d z^{\prime},
$$

which obviously imply

$$
u=-B_{z}, \quad v=A_{z} .
$$

The equation of continuity, when expressed in terms of these functions, becomes

$$
\frac{\partial}{\partial z}\left\{w-B_{x}+A_{y}\right\}=0
$$

and since the velocity is assumed to vanish at infinity,

$$
w=B_{x}-A_{y} \text {. }
$$

It is thus trivial to verify that $A$ is indeed a vector stream potential for which

$$
u=\nabla \times A .
$$

Thus only two scalar functions are needed to specify the velocity field and the equation of continuity will automatically be satisfied by any velocity 
field calculated from them.

The fact that the velocity components $u$ and $v$ are biharmonic functions implies that

$$
\frac{\partial}{\partial z} \nabla^{4} B=0=\frac{\partial}{\partial z} \nabla^{4} A
$$

and incidentally justifies all the changes of orders of differentiations which are used. But the definitions of $A$ and $B$ imply that they both vanish at infinity, so that

$$
\nabla^{4} A=0=\nabla^{4} B
$$

Thus the determination of three biharmonic functions connected together by the continuity equation has been reduced to the determination of two functions unrestricted by the continuity equation. However a coupling restriction between the functions $A$ and $B$ still exists in order to make the corresponding velocity field compatible with an appropriate pressure field. Given an arbitrary velocity field $u$, equation ( 1 ) can be integrated to determine a single valued pressure field which vanishes at infinity, if and only if

$$
\begin{aligned}
0 & =\nabla \times\left\{\nabla^{2} u\right\}=\nabla \times\left\{\nabla^{2}(\nabla \times A)\right\} \\
& =\nabla^{2}\{\nabla \times(\nabla \times A)\} \\
& =\nabla\left\{\nabla^{2}(\nabla \cdot A)\right\},
\end{aligned}
$$

where the fact that each component of $A$ is biharmonic has been used. Since $A$ and its derivatives vanish at infinity it follows that

$$
0=\nabla^{2}(\nabla \cdot A)=\nabla^{2}\left(A_{x}+B_{y}\right) \text {. }
$$

The mathematical problem has thus been reduced to the determination of two biharmonic functions which must in addition satisfy the coupling condition (6). For certain classes of boundary conditions of practical interest it is possible to arrange the calculations so as to avoid the problems implicit in the calculation. Let the boundary conditions be an appropriate selection from among the following cases.

(1) Rigid surface on which the velocities are given on $z=Z(x, y), u=U(x, y), v=V(x, y), w=W(x, y)$.

On such surfaces 


$$
A_{z}=V, B_{z}=-U, B_{x}-A_{y}=W
$$

(2) No-shear surface on a surface $z$ constant. In this case the normal velocity component is given, as are the two tangential components of the disturbance shear stress vector $\mu \nabla \times u$ or $\mu \nabla \times(\nabla \times A)$. Thus the boundary conditions are of the form

$$
\begin{gathered}
B_{x}-A_{y}=W, \\
B_{x y}-A_{y y}-A_{z z}=\mu^{-1 \hat{y z}}, A_{x y}-B_{x x}-B_{z z}=\mu^{-1} \hat{x z},
\end{gathered}
$$

where the symbol $\wedge$ is used to denote a stress.

(3) Plane of symmetry on $z=0$. Here the boundary conditions derive from the fact that the velocity components $u$ and $v$ are even functions of $z$ while $w$ is odd, results which imply

$$
A_{z z}=0, B_{z z}=0, B_{x}-A_{y}=0 \text {. }
$$

It is convenient as a first step towards the solution to ignore the compatibility equation (6) and seek a partial solution which removes the forcing terms from the boundary conditions. Now it is a feature of the introduction of the functions $A$ and $B$ that the above boundary conditions have become much closer to the forms appropriate to the biharmonic equation. Also it is easy to arrange an addition to the boundary conditions so that the problems of determining a pair of biharmonic functions which satisfy all the necessary boundary conditions are not coupled together through these boundary conditions. Thus consider the pair of biharmonic functions $A_{1}$ and $B_{1}$, both vanishing at infinity and which in addition satisfy an appropriate selection from among the following boundary conditions:-

(1) Rigid surface

$$
A_{1 y}=0, A_{1 z}=V ; B_{1 z}=-U ; B_{1 x}=W ;
$$

(2) No-shear surface

$$
A_{1}=0, A_{1 z z}=W_{y}-\mu^{-1} \hat{y z} ; \quad B_{1 x}=W, \quad B_{1 z z}=-W_{x}+\mu^{-1} \hat{x z} ;
$$

(3) Plane of symmetry 


$$
A_{1}=0, A_{1 z z}=0 ; B_{1}=0, B_{1 z z}=0 .
$$

Such a function pair is uniquely determined from standard boundary value problems for the biharmonic equation.

If this pair satisfied the compatibility condition (6) the problem would be solved; but normally this will not happen. Thus the difference functions, $A_{2}$ and $B_{2}$, defined by

$$
A_{2}=A-A_{1}, B_{2}=B-B_{1},
$$

both satisfy the biharmonic equation, the relevant homogeneous boundary conditions and the compatibility condition

$$
\nabla^{2}\left\{A_{2 x}+B_{2 y}\right\}=-\nabla^{2}\left\{A_{1 x}+B_{1 y}\right\}=R \text {, }
$$

where $R$ may be considered as a known function. Thus one is still faced with a pair of coupled biharmonic problems to solve and there might seem little point to the transformation to homogeneous boundary conditions. But for all the boundary conditions under consideration on each portion of the boundary one condition is

$$
B_{2 x}-A_{2 y}=0
$$

and the other two involve the vanishing of the same z-derivatives of $A_{2}$ and $B_{2}$ respectively. The compatibility condition (7) is only on a combination of $A_{2}$ and $B_{2}$. Thus it follows that it is possible to satisfy all the conditions with a solution pair for which

$$
B_{2 x}-A_{2 y} \equiv 0 \text {. }
$$

Thus one does not need to consider a function pair but instead one can work with the single function $F$, which exists, such that

$$
B_{2}=F_{y}, A_{2}=F_{x},
$$

and for which it may be assumed that $F$ vanishes at infinity. Thus this function $F$ satisfies the biharmonic equation, and the compatibility condition

$$
\nabla^{2}\left\{F_{x x}+F_{y y}\right\}=-\nabla^{2}\left\{A_{1 x}+B_{1 y}\right\}
$$

The boundary conditions, when expressed in terms of $F$, will be satisfied 
if one chooses

1) for a rigid surface

$$
F_{z}=0 ;
$$

2) for a no-shear surface

$$
E_{z z}=0
$$

3) for a plane of symmetry

$$
F_{z Z}=0 \text {. }
$$

Now any biharmonic function $E$ which satisfies equation ( 8 ) must also satisfy

$$
\nabla^{2} F_{z z}=\nabla^{2}\left\{A_{1 x}+B_{1 y}\right\}
$$

Thus $\left\{F_{z z}-\left(A_{1 x}+B_{1 y}\right)\right\}$ is a harmonic function, which it is convenient to write as $\Psi_{z}$, so that one can represent

$$
F_{z}=\Psi-\int_{z}^{\infty}\left(A_{1 x}+B_{1 y}\right)
$$

Now if $\Psi$ is any harmonic function vanishing at infinity and $F$ is defined from it by equation (10), then it follows that such an $F$ is biharmonic since $A_{1}$ and $B_{1}$ are biharmonic. Since equation (10) implies equation (9) the function $F$ so generated also satisfies the compatibility condition ( 8$)$. Thus one can determine $F$ by finding a harmonic function $\Psi$, vanishing at infinity and satisfying boundary conditions on $\Psi_{z}$ or $\Psi$ determined from equation (10) and the relevant boundary conditions on $F_{z}$ or $F_{z z}$. In this way the determination of $F$ is reduced to determination of a harmonic function satisfying standard boundary conditions. This organization of the calculations has thus removed the coupling of the boundary conditions which made the original formulation of the problem intractable. Whatever methods one may have to resort to in order to solve the biharmonic and harmonic functions involved, the complexity of the calculations will be reduced by the organization proposed here.

To complete the general calculations one may note that the velocity 
components are given by

$$
\begin{aligned}
& u=-B_{1 z}-\Psi_{y}+\int_{z}^{\infty}\left(A_{1 x y}+B_{1 y y}\right), \\
& v=A_{1 z}+\Psi_{x}-\int_{z}^{\infty}\left(A_{1 x x}+B_{1 x y}\right), \\
& w=B_{1 x}-A_{1 y} .
\end{aligned}
$$

The pressure field can be obtained by integrating any one of the momentum equations from infinity.

\section{Modification of a shear flow by no-shear zone}

Consider a shear flow with a velocity field $\mu^{-1} T(z, 0,0)$ occupying the half space $z>0$. Here we will look at the modification of this flow when there are some no-shear zones on the plane $z=0$. For all subsequent discussion only the disturbance field will be considered and the appropriate boundary conditions for this disturbance velocity field, in any no-shear zone, are

$$
u_{z}=-\mu^{-1}, \quad v_{z}=0, w=0,
$$

and on the remainder of the plane

$$
u=0, v=0, w=0 .
$$

Thus, in the notation of the previous section, $A_{1} \equiv 0$ and the boundary conditions for $B_{1}$ become, on $z=0$,

$$
B_{1}=0, \quad B_{1 z z}=\mu^{-1} T \text { in any no-shear zone }
$$

and

$$
B_{1}=0, B_{12}=0 \text { elsewhere. }
$$

It is apparent that the biharmonic function $z \phi$, where $\phi$ is a harmonic function, solves the problem if

$$
\begin{aligned}
\phi_{z} & =\frac{1}{2} \mu^{-1} T \text { in any no-shear zone, } \\
\phi & =0 \text { els ewhere. }
\end{aligned}
$$


Further simplification can be achieved by using this particular form in equation (9) which becomes

$$
\nabla_{z z}^{2} F^{2}(z \phi)_{y}=2 \phi_{y z} .
$$

The equation

$$
\nabla^{2} F_{z}=2 \phi_{y}
$$

has a particular integral $y \phi$, and so

$$
F_{z}=y \phi+\psi,
$$

where $\psi$ is a harmonic function satisfying the boundary conditions

$$
\begin{aligned}
\psi_{z} & =-\frac{1}{2} \mu^{-1} T y \text { in any no-shear zone, } \\
\psi & =0 \text { elsewhere. }
\end{aligned}
$$

The problem thus devolves to two mixed boundary value problems for Laplace's equations. In terms of these two solutions the complete field may be shown to be described by

$$
\begin{aligned}
& u=-\left\{(z \phi)_{z}+(y \phi)_{y}+\psi_{y}\right\}, \\
& v=y \phi_{x}+\psi_{x}, \\
& w=z \phi_{x}, \\
& p=2 \mu \phi_{x} .
\end{aligned}
$$

It is a trivial matter to verify that these satisfy equations (1) and (2) and will satisfy the boundary conditions.

For a circular no-shear zone the solutions for $\phi$ and $\psi$ are available from work in other contexts, for example, Levey and Wynter [4], and one could compute the disturbance velocity field. However this would not appear to offer any fair basis of comparison of the effects of slots and finite regions of no-shear zones. In any fair comparison the areas should be comparable so that one wants a distribution of finite domains occupying the same fraction of the area of the plane as the slots. Moreover the lack of realism associated with the assumption of uniform shear in the basic flow weighs against heavy calculations. Thus here a 
distribution of no-shear zones of dimension small in comparison with their spacing will be compared with Philip's [5] results for a distribution of parallel slots. This solution predicts a finite value of limu as $z$ tends to infinity and this seems a reasonable measure to adopt for the non-local effect of the no-shear zones. While Philip's answers are not restricted to small area ratios it would require very heavy calculations to obtain the corresponding answers even for the simplest bounded domain.

Consider first a series of similar no-shear zones, of typical dimension $a$, with their centroids at the points $(m l, n l, 0)$ where $m$ and $n$ assume all integer values independently and $l$ is the spacing. Then the small fraction $\varepsilon$ of the area occupied by no shear zones is given by $\pi a^{2} / l^{2}$. Because each zone is relatively far from the other the disturbance shear stress on any zone due to the others should be small and hence as a reasonable first approximation one should be able to merely superpose the fields due to individual zones. To confirm this one may note the form of the boundary conditions for $\phi$ and $\psi$, and using an electrostatic analogy, argue that at large distances $\phi$ behaves as a dipole field and $\psi$ as a quadrupole field. Thus it can be shown that the velocity field due to a single no-shear zone is $O\left(\mu^{-1} \mathrm{Ta}^{3} / \mathrm{r}^{2}\right)$, while the shear stress is $O\left(T a^{3} / r^{3}\right)$. With the fields of each zone superposed the shear stress on any one zone is bounded by $K \Sigma T(a / l)^{3}\left(m^{2}+n^{2}\right)^{-3 / 2}$ which is strictly smaller by $\varepsilon^{3 / 2}$ than the stress which initially had to be removed. Thus for small enough $\varepsilon$ one could in principle continue to reduce the error by repetition and hence to regard the fractional error $O\left(\varepsilon^{3 / 2}\right)$ in the velocity field obtained by superposition.

Let the leading term in the far field expansion for $\phi$ be $-Q z\left(x^{2}+y^{2}+z^{2}\right)^{-3 / 2}$ where $Q$ is a positive constant which depends on the shape of the no-shear zone. Then it may be shown that, for the single $z$ one, the far field velocity component $u$ is given by

$$
u \approx 3 Q x^{2} y\left(x^{2}+y^{2}+z^{2}\right)^{-5 / 2}
$$

When the fields of the individual zones are superposed and one considers $z / 2$ large, 


$$
\begin{aligned}
& u \text { ‡ } 3 Q z \Sigma(m l)^{2}\left(m^{2} l^{2}+n^{2} z^{2}+z^{2}\right)^{-5 / 2} \\
& \forall 3 Q z^{-2} \Sigma(m z / z)^{2}\left\{(m z / z)^{2}+(n z / z)^{2}+1\right\}^{-5 / 2} .
\end{aligned}
$$

If $f(x, y)$ denote $x^{2}\left(x^{2}+y^{2}+1\right)^{-5 / 2}$ then the summation is of the values taken by $f(x, y)$ at intervals $(z / z)$ in both $x$ and $y$. Thus it follows that

$$
\begin{aligned}
\lim _{z \rightarrow \infty} u & =3 Q z^{-2} \iint x^{2}\left(x^{2}+y^{2}+1\right)^{-5 / 2} \\
& =2 \pi Q z^{-2} .
\end{aligned}
$$

It is apparent that the zones do not have to be exactly evenly spaced for this result to apply but they could be distributed statistically provided that the distribution would be such as to permit $\iint f$ to be determined from them. Moreover a similar form of result would apply even if the zones were not identical in their values of $Q$ but had them randomly distributed (for many appropriate probability distributions).

The quantity $Q$ which depends on the particular no-shear zone being considered can only be determined easily for simple regions, but many properties can be established quite simply. If the value of $\phi$ was known on the no-shear zone then the standard representation (Jeffreys and Jeffreys [2])

$$
\phi=(2 \pi)^{-1} z \iint \phi(\xi, n)\left\{(x-\xi)^{2}+(y-n)^{2}+z^{2}\right\}^{-3 / 2} d \xi d \eta
$$

makes it possible to show that

$$
Q=-(2 \pi)^{-1} \iint \phi(\xi, n) d \xi d n \text {. }
$$

By considering the boundary value problem satisfied by $\phi$ it is easy to show by scale transformations that $\mu Q /\left(T a^{3}\right)$ is a function solely of the shape of the no-shear zone $D$. Hence

$$
\lim _{z \rightarrow \infty} u=K(D) T a^{3} /\left(z^{2} \mu\right) \text {. }
$$

Moreover by simple applications of the Maximum Principle for harmonic functions it is possible to show that for two domains $D$ and $D^{\prime}$, with $D \supset D^{\prime}, K(D)>K\left(D^{\prime}\right)$ which provides a means of bounding the values of $K$ 
for awkward shaped domains for which calculations are not easy. If one applies the results available in Levey and Wynter [4] it may be shown that for a circle $K$ takes the value $2 / 3$, which provides a basis for comparison with the corresponding result of Philip [5]. Both results are proportional to $T a \varepsilon / \mu$, where for the slot $a$ is its half-width, and the radius for the circle, but there is a distinct difference in the constant of proportionality. For the circle the constant is $2 /(3 \pi)$ whereas for the slot it is $\pi / 4$. This would suggest that the use of results for slots to estimate the effect of no-shear zones of finite extent may give estimates which are rather large.

\section{References}

[1] T. Brooke Benjamin and J.J. Mahony, "On an invariant property of water waves", J. Fluid Mech. 49 (1971), 385-390.

[2] Harold Jeffreys and Bertha Swirles Jeffreys, Methods of mathematical physics, 3rd ed. (Cambridge University Press, Cambridge, 1956).

[3] Horace Lamb, Hydrodynamics, 6th ed. (Cambridge University Press, Cambridge, 1932).

[4] H.C. Levey and P.E. Wynter, "The circular wing and related problems", Proc. Roy. Soc. Ser. A 268 (1962), 390-404.

[5] J.R. Philip, "Flows satisfying mixed no-slip and no-shear conditions", submitted to 2 . angew. Math. Phys.

[6] W.E. Williams, "Boundary effects in Stokes flows", J. Fluid Mech. 24 (1966), 285-291.

Department of Mathematics,

University of Western Australia,

Nedlands,

Western Australia. 